\title{
MATHEMATICAL MODELLING OF MULTIWAVE VOLUME FREE ELECTRON LASER: BASIC PRINCIPLES AND NUMERICAL EXPERIMENTS
}

\author{
K. BATRAKOV and S. SYTOVA \\ Research Institute for Nuclear Problems, Belarusian State University \\ Bobruiskaya 11, 220050 Minsk, Belarus \\ E-mail: batrakov@inp.minsk.by; sytova@inp.minsk. by
}

Received September 29, 2005; revised January 5, 2006

\begin{abstract}
This contribution is devoted to investigation of multiwave Volume Free Electron Laser (VFEL) by methods of mathematical modelling. Special emphasis is placed on consideration of three-wave VFEL. Mathematical modelling carried out confirmed some preliminary physical estimates. Computer code VOLC for simulation of different schemes of two- and three-wave VFEL is described.

Key words: free electron laser, quasi-Cherenkov instability, simulation, nonlinear integro-differential system
\end{abstract}

\section{Introduction}

At present time FELs (Free Electron Lasers) based on different radiation mechanisms are constructed for a wide wavelength range: from centimeter to ultraviolet [10]. Volume Free Electron Laser (VFEL) based on mechanism of multiwave volume distributed feedback (VDFB) was proposed firstly in [4], theoretically developed in [2] and constructed in 2001 [1]. VDFB allows to reduce significantly starting currents and to tune laser frequency. It provides also mode discrimination in oversized systems (systems with transverse linear sizes essentially exceeding generation wavelength). This gives a possibility for generation of laser beams in large volume regions, distribution of high current beam over large cross-section and reducing of electrical load on laser elements.

This article is devoted to investigation by methods of mathematical modelling of multiwave VFEL. Our previous investigations were devoted to numerical modelling of VFEL with two-wave distributed feedback. Theoretical investigations show the great advantage of different multiwave diffraction geometries and in particular of three-wave diffraction geometry. Generation in 
multiwave distributed feedback geometry has many advantages including lasing in inaccessible for traditional schemes ranges of parameters.

\section{Brief Review of Basic VFEL Operation Principles}

Electron beam with initial electron velocity $\mathbf{u}$ and current density $j$ in VFEL (see Fig. 1) can move close to the target or through the target that is a threedimensional spatially-periodic structure of the length $L$. Under diffraction conditions some strongly coupled waves are generated. Under proper phase conditions electrons of the beam are grouped in a deceleration phase and they produce the stimulated emission. In the case of amplification regime external electromagnetic waves are incident to the target. A regime of oscillator can be produced and this oscillator regime is implemented without external waves.

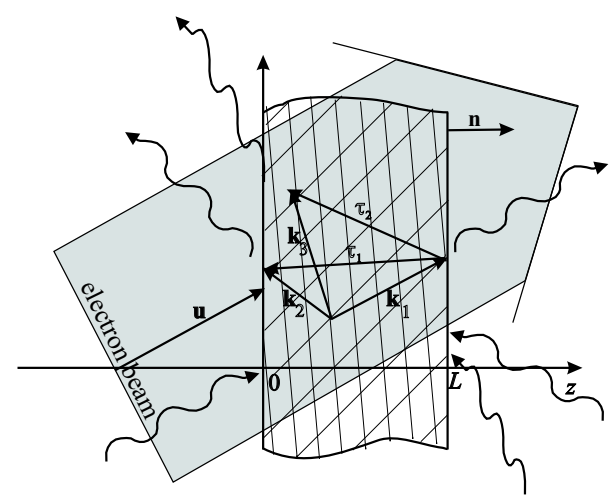

Figure 1. Three-wave VFEL (Bragg-Bragg geometry).

There are three possible geometries in the three-wave system. In the BraggBragg case, depicted in Fig. 1, we deal with the following geometry:

$$
\left(\mathbf{k}_{1}, \mathbf{n}\right)>0, \quad\left(\mathbf{k}_{2}, \mathbf{n}\right)<0, \quad\left(\mathbf{k}_{3}, \mathbf{n}\right)<0,
$$

where $\mathbf{n}$ is a normal vector relative to the surface. The Laue-Laue geometry is implemented when $\left(\mathbf{k}_{1}, \mathbf{n}\right)>0,\left(\mathbf{k}_{2}, \mathbf{n}\right)>0,\left(\mathbf{k}_{3}, \mathbf{n}\right)>0$. We have the BraggLaue geometry, when waves are oriented so that $\left(\mathbf{k}_{1}, \mathbf{n}\right)>0,\left(\mathbf{k}_{2}, \mathbf{n}\right)<0$, $\left(\mathbf{k}_{3}, \mathbf{n}\right)>0$.

\section{Mathematical Model of VFEL}

The system of equations for VFEL is obtained from the Maxwell equations by using the slowly-varying envelope approximation. The dynamics of the electron beam is modelled by averaging over initial phases of electrons. 
The mathematical model describing nonlinear processes developing in three-wave VFEL has the following form:

$$
\begin{aligned}
& \frac{\partial E_{1}}{\partial t}+a_{1} \frac{\partial E_{1}}{\partial z}+b_{11} E_{1}+b_{12} E_{2}+b_{13} E_{3}=I, \quad E_{1}(t, 0)=E_{1}^{0}, \\
& \frac{\partial E_{2}}{\partial t}+a_{2} \frac{\partial E_{2}}{\partial z}+b_{21} E_{1}+b_{22} E_{2}+b_{23} E_{3}=0, \quad E_{2}\left(t, L_{2}\right)=E_{2}^{0}, \\
& \frac{\partial E_{3}}{\partial t}+a_{3} \frac{\partial E_{3}}{\partial z}+b_{31} E_{1}+b_{32} E_{2}+b_{33} E_{3}=0, \quad E_{3}\left(t, L_{3}\right)=E_{3}^{0}, \\
& I=\Phi \int_{0}^{2 \pi} \frac{2 \pi-p}{8 \pi^{2}}(\exp (-i \Theta(t, z, p))+\exp (-i \Theta(t, z,-p))) d p \\
& \frac{d^{2} \Theta(t, z, p)}{d z^{2}}=\Psi\left(k-\frac{d \Theta(t, z, p)}{d z}\right)^{3} \operatorname{Re}\left(E_{1}\left(t-\frac{z}{u}, z\right) \exp (i \Theta(t, z, p)),\right. \\
& \Theta(t, 0, p)=p, \quad \frac{d \Theta(t, 0, p)}{d z}=k-\frac{\omega}{u}, \\
& E_{i}(0, z)=0, \quad i=1,2,3,
\end{aligned}
$$

where $t>0, z \in[0, L], p \in[-2 \pi, 2 \pi]$. We get a system of integro-differential equations with temporal argument $t$, spatial coordinate $z$ and initial electron phase $p$. Amplitudes of electromagnetic field $E_{1}(t, z), E_{2}(t, z), E_{3}(t, z)$ and coefficients $a$ and $b$ are complex-valued, $\Phi$ is imaginary constant. Function $\Theta(t, z, p)$ describes a phase of electron beam relative to electromagnetic wave. $\Theta$ and coefficient $\Psi$ are real valued, $k$ is a projection of the wave vector $\mathbf{k}_{1}$ onto axis $z, \omega$ is a field frequency. Values of boundaries $L_{2}$ and $L_{3}$ for wave vectors $\mathbf{k}_{2}$ and $\mathbf{k}_{3}$ are equal to 0 or $L$ depending on a specific geometry considered in applications. We suppose that all functions are smooth, bounded and slowly varying.

Let us formulate the system of equations and boundary conditions for a general $n$-wave distributed feedback geometry:

$$
\frac{\partial \mathbf{E}}{\partial t}+\mathbf{A} \frac{\partial \mathbf{E}}{\partial z}+\mathbf{B E}=\mathbf{G}(I),
$$

where $\mathbf{E}=\left(E_{1}, E_{2}, \ldots, E_{n}\right)^{T}$. Boundary conditions are described in the following form:

$$
E_{i}\left(t, L_{i}\right)=E_{i}^{0}, \quad i=1, \ldots, n .
$$

Diagonal matrix A contains direction cosines of wave vectors $\mathbf{k}_{i}$. Matrix $\mathbf{B}$ describes dynamical diffraction in the system. $E_{i}^{0}$ are amplitudes of the external incident electromagnetic waves. If $E_{i}^{0}=0$ for all $i$, we deal with oscillator regime. The vector of right-hand sides $\mathbf{G}$ contains components $I$ for waves that are in synchronism with electron beam and are equal to zero for the other waves. 


\section{Numerical Algorithms}

Numerical methods to solve the system (3.5), (3.6), (3.2) - (3.4) are similar to methods proposed in $[6,7]$ for simulation of two-wave VFEL.

Let us introduce in domain $\Omega=\{0 \leq z \leq L,-2 \pi \leq p \leq 2 \pi\} \bigcup\{t>0\}$ uniform grids:

$$
\begin{aligned}
& \omega_{z}=\left\{z_{m}: z_{m}=m h_{z}, m=0,1, \ldots, M, M h_{z}=L\right\}, \\
& \omega_{p}=\left\{p_{j}: \quad p_{j}=h_{p} j, \quad j=-N, \ldots,-1,0,1, \ldots, N, h_{p} N=2 \pi\right\}, \\
& \omega_{t}=\left\{t_{l}: \quad t_{l}=l h_{t}, \quad l=0,1, \ldots\right\} .
\end{aligned}
$$

Discrete functions are defined on the grid $\omega_{t} \times \omega_{z} \times \omega_{p}$, they are denoted by

$$
E_{m}^{l}=E\left(t_{l}, z_{m}\right), \quad \Theta_{m, j}^{l}=\Theta\left(t_{l}, z_{m}, p_{j}\right) .
$$

We approximate the differential problem (3.2), (3.5) by the following finitedifference scheme:

$$
\begin{aligned}
& \frac{\Theta_{m+1, j}^{l+1}-2 \Theta_{m, j}^{l+1}+\Theta_{m-1, j}^{l+1}}{h_{z}^{2}}=\Psi\left(k-\frac{\Theta_{m+1, j}^{l+1}-\Theta_{m-1, j}^{l+1}}{2 h_{z}}\right)^{3} \operatorname{Re}\left(\widetilde{E}_{m}^{l} \exp \left(i \Theta_{m, j}^{l+1}\right)\right), \\
& \frac{\mathbf{E}_{m}^{l+1}-\mathbf{E}_{m}^{l}}{h_{t}}+\mathbf{A C} \frac{\mathbf{E}_{m}^{l+1}-\mathbf{E}_{m \pm 1}^{l+1}}{h_{z}}+\mathbf{B} \frac{\mathbf{E}_{m}^{l+1}+\mathbf{E}_{m \pm 1}^{l+1}}{2}=\mathbf{G}(\mathrm{I}), \\
& \mathrm{I}=\Phi \sum_{j=0}^{N} c_{j}\left(\exp \left(-i \Theta_{m, j}^{l+1}\right)+\exp \left(-i \Theta_{m,-j}^{l+1}\right)\right),
\end{aligned}
$$

where $\widetilde{E}_{m}^{l}=E\left(t_{l}-\alpha t_{m}, z_{m}\right), \alpha=h_{z} /\left(h_{t} u\right)$. We take the integer part of $\alpha$. When $t_{l}-\alpha t_{m}<0$ we assume that $\widetilde{E}=E\left(0, z_{m}\right) . c_{j}$ are coefficients of quadrature formula and we use the trapezoidal rule for approximation of the integral.

The convection term is approximated by the upwind finite-difference formula $\mathbf{C} \frac{\mathbf{E}_{m}^{l+1}-\mathbf{E}_{m \pm 1}^{l+1}}{h_{z}}$. We choose a proper right or left finite-difference derivative with respect to $z$ in dependence of the wave spread direction. Here $\mathbf{C}$ is a diagonal matrix with coefficients equal to " +1 " or "-1", corresponding to the left or right finite-difference derivative respectively for each wave amplitude $E_{1}, E_{2}, \ldots, E_{n}$ (see e.g. (2.1)). Let consider the example of two-wave VFEL, when wave with amplitude $E_{1}$ goes from the left boundary to the right one and $E_{2}$ goes in opposite direction (that is $A_{11}>0, A_{22}<0$ ). Then system (4.2) has the following form:

$$
\begin{aligned}
\frac{E_{1, m}^{l+1}-E_{1, m}^{l}}{h_{t}}+A_{11} \frac{E_{1, m}^{l+1}-E_{1, m-1}^{l+1}}{h_{z}} & +B_{11} \frac{E_{1, m}^{l+1}+E_{1, m-1}^{l+1}}{2} \\
& +B_{12} \frac{E_{2, m}^{l+1}+E_{2, m-1}^{l+1}}{2}=\mathrm{I},
\end{aligned}
$$




$$
\begin{aligned}
\frac{E_{2, m}^{l+1}-E_{2, m}^{l}}{h_{t}}-A_{22} \frac{E_{2, m}^{l+1}-E_{2, m+1}^{l+1}}{h_{z}} & +B_{21} \frac{E_{1, m}^{l+1}+E_{1, m+1}^{l+1}}{2} \\
& +B_{22} \frac{E_{2, m}^{l+1}+E_{2, m+1}^{l+1}}{2}=0 .
\end{aligned}
$$

Investigation of stability of difference scheme (4.1)-(4.2) is a very difficult problem, thus we restrict ourselves to mark that the linearized problem is obviously stable with respect to initial conditions and right-hand sides. Stability of the full system (4.1)-(4.2) was confirmed in many numerical experiments.

Equation (4.1) is solved by using the Picard type iterative process as it was proposed in [5] and the number of required inner iterations is small.

We note that the proposed algorithm allows us to use parallel processing. The main work content is the computation of $\Theta_{m+1, j}^{l+1}$ in (4.1). Since all calculations are independent with respect to $j$, so all $\Theta_{m+1, j}^{l+1}$ can be computed in parallel on standard SMP computing system, where processors use a shared memory.

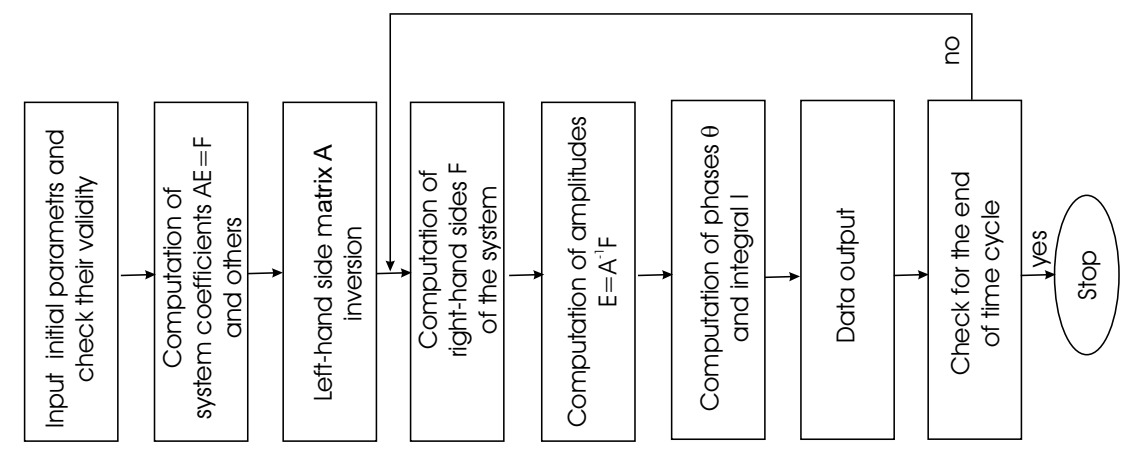

Figure 2. Block-scheme of VOLC.

\section{Computer Code VOLC}

There are a lot of different computer codes for modelling FEL [9]. The successful FEL operation depends on a large number of input parameters. It is clear that only computer simulations allow us to choose the optimal set of parameters in order to obtain the greater radiation output. In [9] a comparison of different existing FEL code with respect to dimensionality, time-dependents of simulation, type of model for description of particle beam is presented. It is shown that a beam description by collective variables allows the faster integration in time than the method of Particle-in-Cell.

Computer code VOLC that means VOLume Code was developed on the basis of multiple Fortran codes, created in 1991-2005 years. This code implements different geometries of two- and three-wave VFEL (i.e. $n=2$ or $n=3$ 
in (3.5)). In the future we plan to expand the possibility of VOLC up to $n=6$. The beam is modelled by averaging over initial phases of electrons that means by collective variables. Dimensionality is $2 \mathrm{D}$ (one spatial coordinate and one phase space coordinate) plus time. A block-scheme of VOLC is presented in Fig. 2. Its interface is presented in Fig. 3.

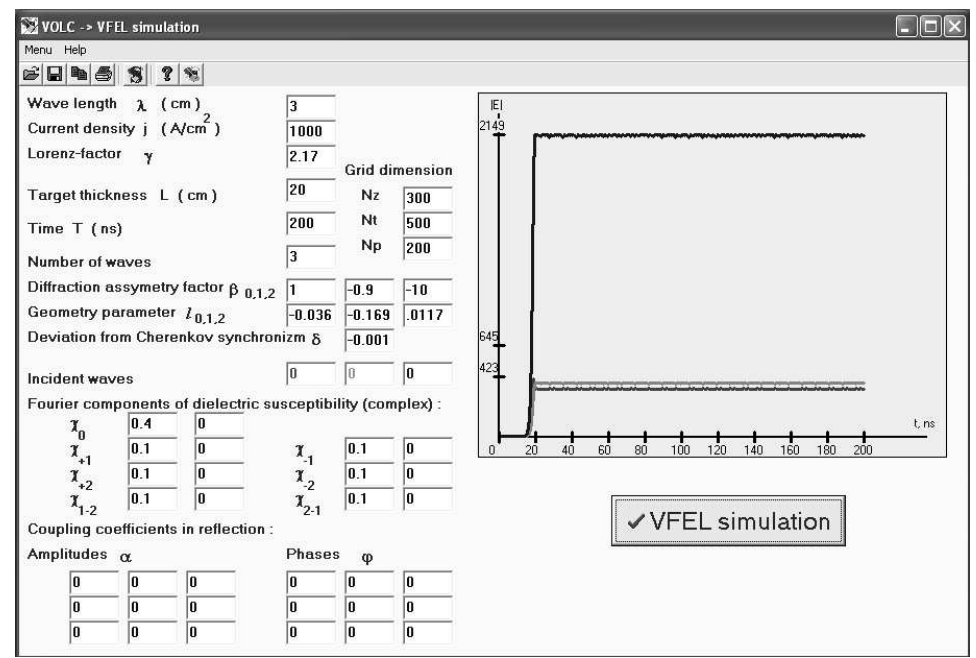

Figure 3. Interface of computer code VOLC.

\section{Results of Numerical Experiments}

VOLC was tested with carping. Different regimes, such as oscillator and amplifier regimes, SASE (Self-Amplified Spontaneous Emission), BWT (backward wave tube), TWT (travelling wave tube), BWT-TWT were investigated. All results correspond well to predictions of physical theory.

In this section we present some results of numerical experiments carried out using code VOLC. Different regimes of three-wave VFEL operation were investigated. We obtained various examples of establishment of nonstationary solutions, including steady-state smooth solutions, oscillations, as well as chaotic regimes. The stationary regime is realized when the beam current exceeds some threshold value. Further increase of beam current density leads to establishing of different periodic regimes. In Fig. 4 the periodic regime of VFEL intensity in the Bragg-Bragg and the corresponding phase space portrait are given.

It is evident that after establishing of asymptotic regime we deal with periodic $1 \mathrm{~T}$ and $5 \mathrm{~T}$ regimes, perturbed with some computational noise. This is illustrated in Fig. 5 and Fig. 6. 


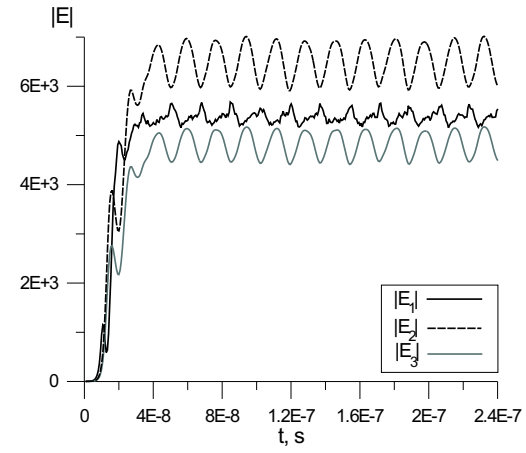

a)

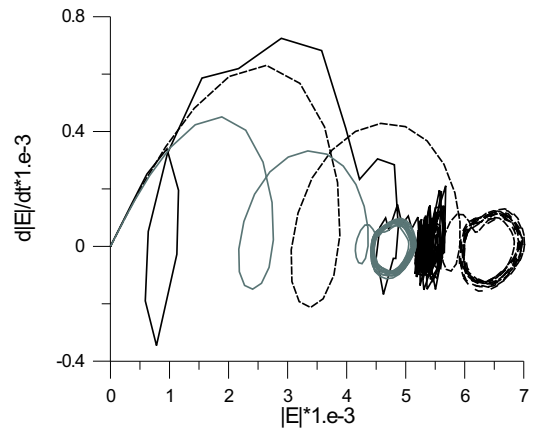

b)

Figure 4. Periodic regimes of VFEL: (a) intensity, (b) the corresponding phase space portrait.

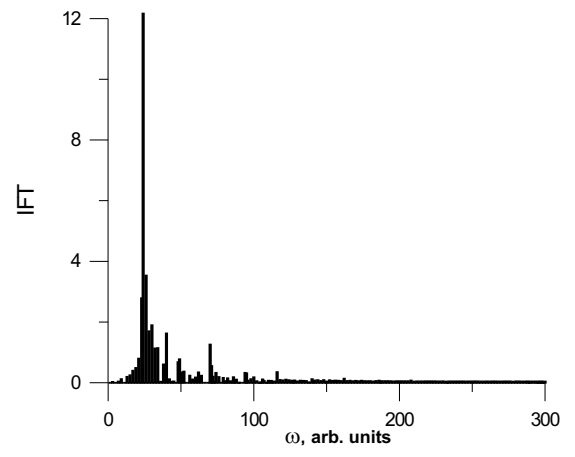

a)

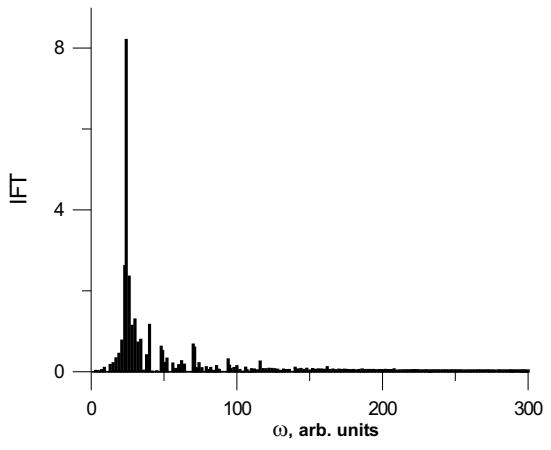

b)

Figure 5. Fourier $1 \mathrm{~T}$ periodic regimes corresponding to the amplitudes: (a) $E_{2}$, (b) $E_{3}$ from Fig. 4 .

One of the important parameters in VFEL operating is the current threshold $j_{t h}$. It is a minimal current density at which the process of generation begins. The possibility to reduce the current threshold is one of the main advantages of VFEL as compared with the other generators of electromagnetic energy. This reduction is attained due to the fact that VFEL allows to have some modes in synchronism. This is one of the main VFEL features since synchronism suppresses parasitic modes in the system. If only one mode is in synchronism with the electron beam, the threshold current $j_{t h} \sim \frac{1}{(k L)^{3}}$, where $k=\omega / c$ (see [3]). If two modes are in synchronism with electrons, then we have the estimate $j_{t h} \sim \frac{1}{(k L)^{5}}$, and in general case when $n$ modes are in synchronism with electrons, then $j_{t h} \sim \frac{1}{(k L)^{3+2(n-1)}}$. 


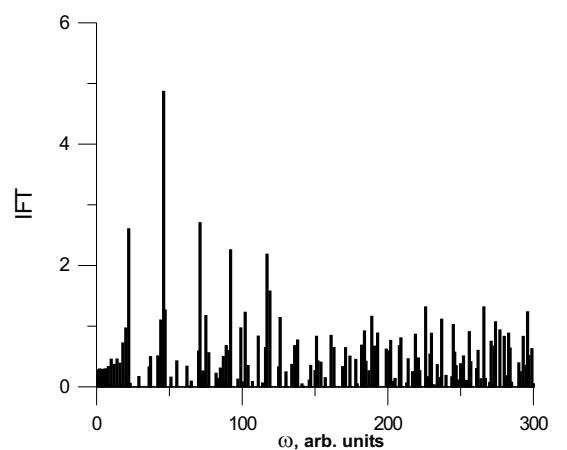

a)

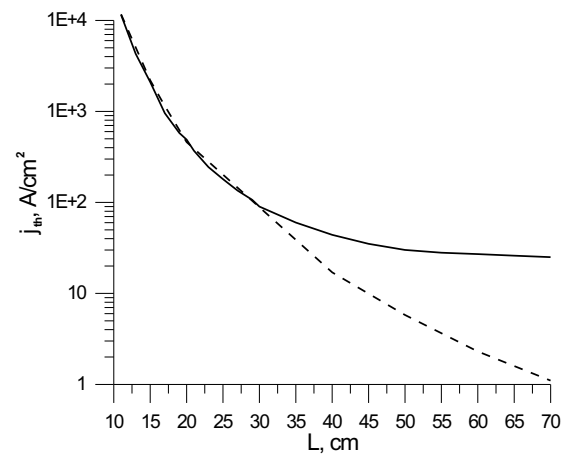

b)

Figure 6. (a) Fourier $5 \mathrm{~T}$ periodic regime corresponding to amplitude $E_{1}$ from Fig. 4, (b) dependence of the current threshold $j_{t h}$ on length of the target $L$ for two-wave VFEL (solid line) and three-wave VFEL (dashed line).

Thus the threshold current can be significantly decreased when modes are degenerated in multiwave diffraction geometry. On the other hand interaction length can be reduced at a given current value.

The theoretical results were confirmed by numerical experiments. In Fig. $6 \mathrm{~b}$ the comparison of dependence of current threshold $j_{t h}$ on the length of the target $L$ is demonstrated for two- and three-wave geometry. This is a good illustration of effectiveness of volume distributed feedback. It is evident that the threshold current can be significantly decreased in multiwave diffraction geometry.

Synchronism of several modes with electrons corresponds to roots degeneration of dispersion equation. As it was mentioned above, the necessary condition for VFEL operation is the execution of diffraction conditions [8]. For this the initial parameters should be chosen close to the point of degeneration of dispersion equation. For system (3.5) the root degeneration is selected from the following equation:

$$
\operatorname{det}(\mathbf{B})=0
$$

where $\mathbf{B}=\mathbf{B}\left(k_{1}\right), k_{1}$ is a projection of wave vector $\mathbf{k}_{1}$ on axis z. We did not write explicitly all coefficients of the system (3.5) here. In the two-root degeneration mode the additional condition

$$
\operatorname{det}\left(\frac{\partial \mathbf{B}}{\partial k_{1}}\right)=0
$$

should be fulfilled together with (6.1). The following extra equation

$$
\operatorname{det}\left(\frac{\partial^{2} \mathbf{B}}{\partial k_{1}^{2}}\right)=0
$$

corresponds to generation in three-root degeneration mode. Each next equation narrows the domain of permissible parameters and allows to obtain more 


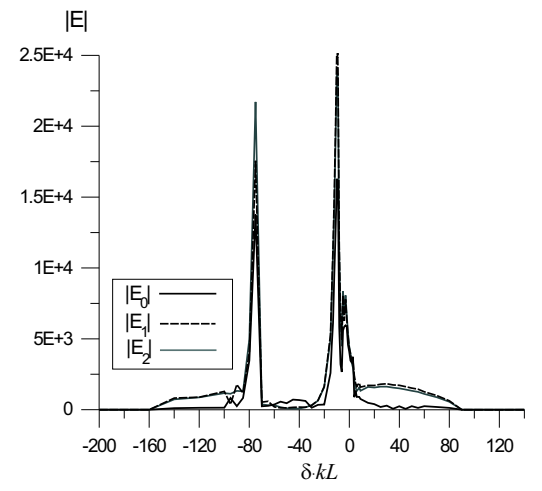

a)

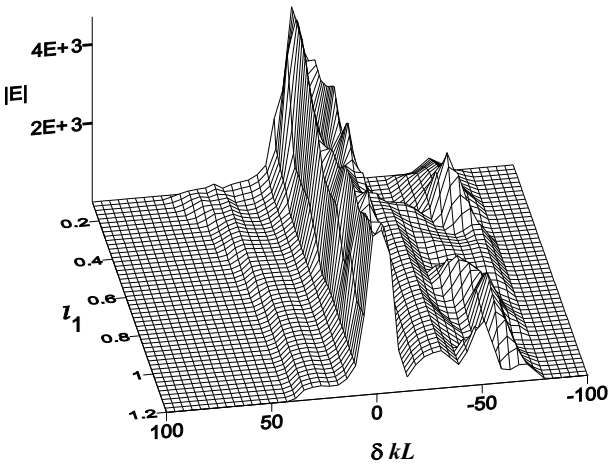

b)

Figure 7. One mode synchronism case: (a) dependence on detuning from exact Cherenkov condition $\delta$ (arbitrary units), (b) dependence on $\delta$ and system parameter $l_{1}$ for amplitude $E_{1}$ in one-mode synchronism.

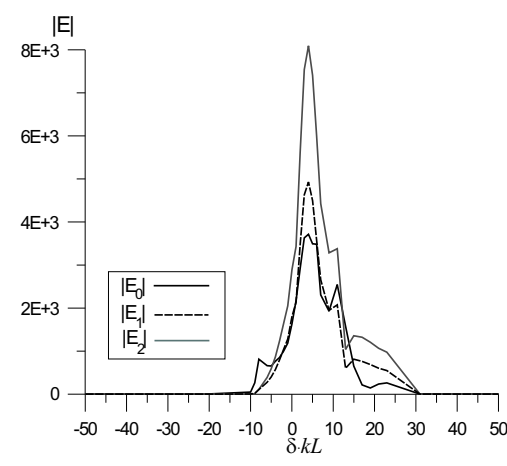

a)

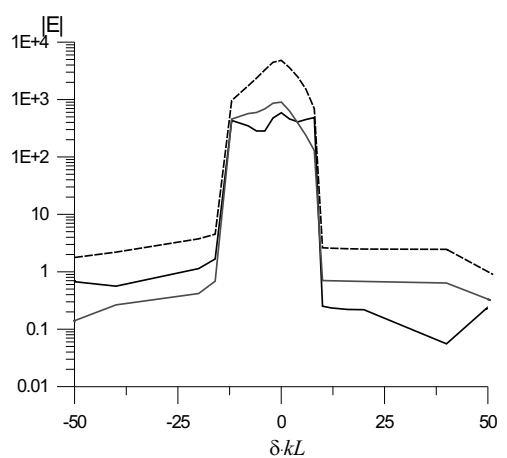

b)

Figure 8. (a) Two-root degeneration case, (b) three-root degeneration case.

radiation at smaller beam current. This was confirmed in numerical experiments and some results are presented in Fig. 7 and Fig. 8. So, if only one mode is in synchronism (i.e. system parameters satisfy only the first dispersion equation) one can see dependence on so-called detuning from the exact Cherenkov condition $\delta$. Three peaks correspond to three possible roots (Fig. 7a). In Fig. 7b we present an example of dependence of amplitude $E_{1}$ on $\delta$ and one of the system parameters $l_{1}$. Previous figure is a normal cut at fixed value of $l_{1}$. The picture in Fig. $8 \mathrm{a}$ presents two-root degeneration case with two peaks. And the last figure Fig. $8 \mathrm{~b}$ shows three-root degeneration case with one peak corresponding to the single solution of the system of dispersion equations $(6.1)-(6.3)$. 


\section{Conclusions}

Mathematical models and computer code VOLC described here can be used effectively in modelling of nonlinear regimes of VFEL operation. They will be useful for providing experiments on VFEL on the installations created at the Research Institute for Nuclear Problems of Belarusian State University. Authors thank Prof. Baryshevsky for permanent interest to their work.

\section{References}

[1] V. Baryshevsky, K. Batrakov and al. First lasing of a volume FEL (VFEL) at a wavelength range 4-6 mm. Nucl. Instr. and Meth. in Phys. Res., A483, 21-24, 2002.

[2] V. Baryshevsky, K. Batrakov and I. Dubovskaya. Parametric (quasi-Cherenkov) X-ray FEL. Journ.Phys.D, 24, 1250-1257, 1991.

[3] V. Baryshevsky, K. Batrakov and I. Dubovskaya. Formation of distributed feedback in an fel under multi-wave diffraction. Nucl. Instr. and Meth. in Phys. Res., A358, 493-496, 1995.

[4] V. Baryshevsky and I. Feranchuk. Parametric beam instability of relativistic charged particles in a crystal. Phys.Let.A, 102, 141-144, 1984.

[5] K. Batrakov and S. Sytova. Modelling of quasi-Cherenkov electron beam instability in periodical structures. Mathematical Modelling and Analysis, 9(1), $1-8,2004$.

[6] K. Batrakov and S. Sytova. Modelling of Volume Free Electron Lasers. Computational Mathematics and Mathematical Physics, 45(4), 666-676, 2005.

[7] K. Batrakov and S. Sytova. Nonstationary stage of quasi-Cherenkov beam instability in periodical structure. Mathematical Modelling and Analysis, 10(1), $1-8,2005$.

[8] SL. Chang. Multiple Diffraction of X-ray in Crystals. Springer-Verlag, Berlin, Heidelberg, New. York, Tokyo, 1984.

[9] S. Reiche. Computation of FEL processes. In: J.Chew, P.Lucas and S.Webber(Eds.), Proc. of the 2003 Particle Accelerator Conference, Trieste, Italy, 2003, IEEE, Portland, Oregon, USA, 203 - 207, 2003.

[10] W.B.Colson and B.W.Williams. Free Electron Lasers in 2004. In: R. Baker et al.(Ed.), Proc. of the 26th Intern. Free Electron Laser Conference FEL2004, Trieste, Italy, 2004, Comitato Conferenze Elettra, Trieste/Italy, 706 - 710, 2004 . 